\title{
Segregación espacial, satisfacción con el barrio y comportamientos responsables con el medio ambiente en una ciudad latinoamericana
}

\author{
Segregação espacial, satisfação com o bairro e \\ comportamento responsável com o meio ambiente em uma \\ cidade latino-americana \\ Spatial segregation, neighborhood satisfaction, and \\ environmentally responsible behavior in a Latin American city
}

Paola García-Reyes [a] (iD), Natalia Andrea-Hernández-Vargas [b] (iD),

Ciro David Murillo-Lenis [a] (iD)

[a] Universidad del Norte de Colombia, Departamento de Ciencia Política y Relaciones Internacionales, Barranquilla, Colombia

[b] Universidad del Norte de Colombia, Barranquilla, Colombia

Cómo citar: García-Reyes, P., Hernández-Vargas, N. A., \& Murillo-Lenis, C. D. (2021). Segregación espacial, satisfacción con el barrio y comportamientos responsables con el medio ambiente en una ciudad latinoamericana. urbe. Revista Brasileira de Gestão Urbana, v. 13, e20210043. https://doi.org/10.1590/2175-3369.013.e20210043

\section{Resumen}

Las urbes latinoamericanas están segregadas en el espacio de forma tal que los ciudadanos de ingresos altos suelen habitar en entornos más seguros, mejor urbanizados y con mejor acceso a servicios. Además, enfrentan problemas ambientales propios de las grandes aglomeraciones urbanas, tales como desequilibrios en la disponibilidad y demanda de agua potable, sobregeneración de residuos sólidos y deficiencias en su tratamiento. En este artículo nos preguntamos por la relación entre la segregación espacial, la satisfacción con el entorno y los comportamientos responsables con el medio ambiente, con base en un análisis multivariado de los datos de la Encuesta de Percepción Ciudadana 2017 aplicada en la ciudad de Barranquilla, Colombia. Los resultados sustentan la existencia de una relación entre los segregación, satisfacción y comportamientos y sugieren dos matices importantes relacionados con los comportamientos que suceden en los espacios privados o públicos, de un lado y con la relación de propiedad o arriendo de las viviendas, de otro.

Palabras clave: Segregación espacial. Satisfacción. Barrio. Medio ambiente.

\section{Resumo}

As urbes latino-americanas são segregadas espacialmente, os cidadãos de ingressos altos costumam habitar nos entornos com maior segurança, melhor urbanizados e com melhor acesso a serviços. Além do mais, enfrentam problemas ambientais próprios das grandes aglomerações urbanas, tais como desequilíbrios na disponibilidade e 
demanda de água potável, superprodução de resíduos sólidos e deficiências no seu tratamento. Este artigo nos perguntamos pela relação entre segregação espacial, satisfação com o entorno e os comportamentos responsáveis com o meio ambiente, com base em uma análise multivariada dos dados da Enquete de Perceção Cidadã 2017, para a cidade de Barranquilla na Colômbia. Os resultados apoiam a existência de uma relação entre segregação, satisfação e comportamentos e sugerem duas nuances importantes relacionadas com os comportamentos que ocorrem em espaços privados ou públicos, por um lado, e com a relação de propriedade ou arrendamento de casas, por outro.

Palavras-chave: Segregação espacial. Satisfação Bairro. Meio ambiente.

\section{Abstract}

Latin American cities are segregated in space in ways that high-income citizens tend to live in safer, betterurbanized neighborhoods, with better access to services. Also, they face environmental problems that are typical of large cities, such as imbalances in the availability and demand of drinking water, over-generation of solid waste, and deficiencies in their treatment. In this article we ask about the relationship between spatial segregation, satisfaction with the neighborhood and responsible behavior with the environment, based on a multivariate analysis of the data of the Survey of Citizen Perception 2017, for Barranquilla city in Colombia. The results support the existence of a relationship between segregation, satisfaction and behaviors and suggest two important nuances related to the behaviors that occur in private or public spaces, on the one hand, and with the relationship of ownership or rental of homes, on the other.

Keywords: Spatial segregation. Satisfaction. Neighborhood. Environment.

\section{Introducción}

En este artículo nos preguntamos por la relación entre segregación espacial, satisfacción con el entorno y comportamientos responsables con el medio ambiente en una ciudad de América Latina. Entendemos esos comportamientos como aquellos que reducen el impacto humano sobre el entorno tales como apagar las luces, reciclar o utilizar medios sostenibles de desplazamiento (Reid et al., 2010; Stern et al. 1999; Xu \& Han, 2019).

América Latina es la región más urbanizada y más desigual del mundo. Este hecho se traduce en una distribución inequitativa del acceso a servicios y beneficios en las ciudades, en exclusión espacial y en la distinta exposición a los riesgos medioambientales (ECLCAC, 2016), algo que Bittencourt y Faria (2021) denominan "el problema de la territorialización de las desigualdades". Los ciudadanos de ingresos altos suelen habitar en entornos más seguros, mejor urbanizados y con buen acceso a vías, parques, servicios públicos y centros de empleo, mientras que los de ingresos bajos habitan en viviendas con equipamientos precarios, ubicadas en entornos menos seguros y con vías de acceso deficientes (Yunda, 2019). Este patrón se ha venido profundizando a partir de la década de 1980 con el aumento en la oferta de desarrollos suburbanos y con la configuración de un estilo de vida asociado a una "internacionalización de los gustos", al fortalecimiento de una cultura del automóvil y al surgimiento de conjuntos habitacionales cerrados (Gilbert \& Ferguson, 1994; Thibert \& Osorio, 2014).

En cuanto a los problemas medioambientales, la población urbana en los países latinoamericanos constituye cerca del $80 \%$ de su población total y se espera que esta proporción aumente en un $10 \%$ más para 2030, con las ciudades intermedias (300.000 - 500.000 habitantes) experimentando los mayores crecimientos (ECLCAC, 2016; Nathaniel et al., 2020). En este escenario, muchas ciudades enfrentan problemas que sobrepasan su capacidad de adecuar sus infraestructuras, tales como asentamientos informales, cambios demográficos, inequidad social o contaminación de fuentes hídricas (Bai et al., 2017). La urbanización contribuye de forma importante a las emisiones de $\mathrm{CO} 2$, las cuales se deben principalmente a procesos de combustión en industrias y al uso de automóviles y calefacciones en los hogares (Montero \& García, 2017; Nathaniel et al., 2020). Si bien la cobertura de agua potable era del 97\% en 1995, la oferta de 
un servicio seguro y adecuado podría llegar a ser entre un 15\% y un 20\% menor, dado que muchos cuerpos de agua se encuentran contaminados (Montero \& García, 2017). Un problema mayor es el de la generación y disposición de residuos sólidos, ya que cerca del $45 \%$ de éstos terminan en vertederos al aire libre, sin separación previa. Este hecho se asocia a problemas medioambientales mayores como contaminación de aguas por lixiviados, contaminación de aire por quema y liberación de metano por descomposición (Bai et al., 2017).

Nos aproximamos a esta relación desde una doble mira: espacial y perceptual. Adoptar una perspectiva espacial para analizar las actitudes frente al ambiente resulta útil en tanto que los hogares ubicados en las mismas comunidades y barrios suelen tener patrones de conducta similares (Brown, 2014). De otra parte, si bien las investigaciones han mostrado que la satisfacción con el entorno está asociada de forma positiva con comportamientos ambientales más responsables (Crociata et al., 2016; Permentier et al., 2010; Ramkissoon et al., 2013), diversos análisis han mostrado que la percepción sobre las condiciones del entorno tiene más peso sobre la satisfacción que las condiciones objetivas mismas (Permentier et al., 2010), por lo que resulta relevante indagar sobre aquellas de forma específica. Entender las formas en que los comportamientos se agrupan y en que resultan motivados, aporta a la comprensión de un campo que ha mostrado ser muy elusivo (Brown, 2014) y otorga herramientas para enforcar las políticas a grupos específicos.

Para responder a esta pregunta nos basamos en el caso de la ciudad de Barranquilla, Colombia, analizando datos de la Encuesta de Percepción Ciudadana, aplicada por la organización Barranquilla Cómo Vamos en 2017, mediante el uso de técnicas estadísticas multivariadas. Estas técnicas permiten describir el comportamiento de un gran número de variables de manera integral y simultánea. Es una práctica que complementa el análisis tradicional de revisar cada característica aisladamente (análisis univariado) o su interacción con otro factor (análisis bivariado), al integrar los resultados de todas las variables. Esto resulta particularmente útil en el análisis de encuestas de percepciones y creencias, donde se presenta que la escala de medida en la que son registradas las respuestas es nominal u ordinal, lo cual impide la implementación de técnicas estadísticas que se basen en variables cuantitativas. Procedemos así: en primer lugar, presentamos una visión de las discusiones de la literatura sobre los temas propuestos. Luego, ilustramos los patrones de segregación presentes en la ciudad. Sobre esta base, recogemos la metodología utilizada en el análisis, así como los resultados de la investigación. En una última sección proponemos un conjunto de conclusiones al respecto.

\section{Espacio, medio ambiente y comportamientos}

La distribución de la población de las ciudades en su espacio físico es resultado de procesos generales de diferenciación (Saraví, 2008) que están influidos por factores institucionales y sociales (Ruiz-Tagle, 2015). En cuanto a los primeros, las políticas gubernamentales impactan las formas en las que se despliegan las ciudades en el espacio y en que son habitadas por sus ciudadanos (Bittencourt \& Faria, 2021). Este hecho fue señalado por Bourdieu (2007) para el caso francés. Según su argumento el Estado con sus políticas habitacionales había llevado a cabo una "verdadera construcción política del espacio, en la media en que favoreció la construcción de grupos homogéneos con fundamento espacial" (p. 124, énfasis en el original). En cuanto a los factores sociales, Saraví (2008) ha señalado con acierto que la estructura del espacio urbano no puede ser entendida si no se la interpreta como la distribución en el espacio de actores socialmente posicionados.

Podemos citar algunos ejemplos de la doble influencia de factores institucionales y sociales sobre la configuración espacial de las ciudades para el caso latinoamericano. En Bogotá, la mayoría de las licencias de construcción de viviendas, oficinas y comercio otorgadas en el período 2010-2015 se concentraron en el sector nororiental, donde habitaban las personas de mayores ingresos. Según Yunda (2019), este hecho evidencia un patrón de desarrollo en el cual la inversión privada fue dirigida por el sistema de estratificación para el cobro de servicios públicos, instituido en Colombia durante la década de 1970. En Curitiba, ciudad vista como modelo de planeación en la región, los recursos en infraestructura invertidos entre 2005 y 2016, terminaron por reproducir 
un modelo espacial en el que la ausencia de esas inversiones indican, no una ausencia sino una acción específica del Estado, que reproduce las desventajas de los sectores periféricos de la ciudad (Bittencourt \& Faria, 2021). En Argentina, los procesos de suburbanización y heterogeneización del territorio del Gran Mendoza fueron resultado de las intervenciones estatales en construcción de vías de acceso y vivienda, las cuales profundizaron la división social del espacio, con la delimitación de áreas residenciales para diferentes clases sociales. Este hecho sumó a la configuración espacial comprensiones sociales acerca del lugares de residencia como deseables o indeseables, contribuyó a la estigmatización de sus pobladores y creó sentidos de pertenencia (Saraví, 2008; Tosoni, 2011).

Esa confluencia de políticas públicas y de orientaciones sociales ha dado forma a patrones de segregación específicos para la región, donde la ocupación del espacio se ha caracterizado por la localización de las personas de bajos recursos en la periferia y la de estratos de ingresos altos en el área céntrica o conectada con ella. Este patrón no se reduce a las residencias, sino que tiene efectos en la aglomeración de empleos y servicios (Rodríguez, 2008). En Ciudad de México, por ejemplo, los sectores de bajos ingresos, si bien se concentran en áreas homogéneamente pobres, trabajan en zonas de la ciudad habitadas o cercanas a las de los sectores de mayores ingresos (Saraví, 2008). Este monocentrismo "acomodado" sobrecarga a los pobres y encapsula a los ricos cerca de sus empleos y lugares de residencia (Rodríguez, 2008).

La revisión de los estudios sobre la relación entre a segregación espacial y los comportamientos proambientales muestra que éstos hechos se relacionan de maneras ambiguas y que esta relación puede estar mediada por la satisfacción con el entorno. La literatura indica que las características socioeconómicas de los hogares están correlacionadas espacialmente y que la interacción al interior y entre comunidades de intereses locales como el barrio, genera, media y propaga comportamientos proambientales asociados al interés colectivo y a la identidad social compartida (Crociata et al., 2016; Reid et al., 2010). No obstante, el aumento en los ingresos ha mostrado tener efectos uniformemente negativos sobre los comportamientos proambientales (Brown, 2014). De otra parte, los atributos específicos del barrio, tales como servicios públicos, aspecto o seguridad parecen estar relacionados con la satisfacción general del barrio y vivir en barrios de mejores ingresos se asocia a mayores niveles de satisfacción (Permentier et al., 2010). A su vez, la satisfacción con el entorno parece estar positivamente asociada con los comportamientos responsables con el medio ambiente, en particular los de bajo costo. Sin embargo, es la percepción del entorno, más que sus atributos objetivos la que predice mejor la satisfacción (Ramkissoon et al., 2013).

Lo anterior implica que los atributos objetivos del entorno solo tienen un efecto indirecto sobre los comportamientos analizados y que éstos tienen connotaciones sociales y culturales. En efecto, la segregación espacial urbana se vincula con lo que la literatura ha dado en llamar "efectos de barrio", "efectos de vecindario" (Ruiz-Tagle, 2015) o "efecto de lugar" (Bourdieu, 2007). Estos efectos pueden ser entendidos como una manifestación de patrones más amplios en los cuáles las regularidades en los territorios tienen consecuencias para las oportunidades, comportamientos y percepciones de quienes los habitan (Borges \& Carvalho, 2017; Delamaza \& Thayer, 2016). Algunos estudios muestran que el área de residencia tiene consecuencias sobre la estigmatización, el empleo y la salud, incluida la salud mental (Kearns \& Parkinson, 2001). Esto es así porque los barrios se constituyen en redes locales de información, en centros de actividades y en paisaje de oportunidades sociales y económicas (Ellen \& Turner, 1997; Galster \& Killen, 1995; Kearns \& Parkinson, 2001). De otra parte, una de las funciones principales de los barrios es la de la de configuración de identidades y la incorporación de valores, normas y comportamientos (Tosoni, 2011).

En este escenario, esta investigación se ubica en lo que Reid, Sutton y Hunter (2010) denominan escala de observación, aproximación pertinente para enmarcar cuestiones sobre las cuáles no hay conocimiento establecido. Nuestro interés primordial es el de indagar por esa relación mediante el uso técnicas estadísticas multivariadas típicamente descriptivas, como un paso previo para el análisis de tipo explicativo. En otras palabras, nos concentramos en el contexto de descubrimiento antes que en el de verificación (Reichenbach, 2006). En lo que sigue, ofrecemos una descripción de la ciudad de Barranquilla y sus patrones de segregación. Luego, presentamos nuestra aproximación analítica y sus resultados y ofrecemos una discusión al respecto. 


\section{Barranquilla, medio ambiente y segregación espacial}

Barranquilla, ciudad ubicada en la costa Caribe colombiana, es por población (1.230.000 habitantes) la cuarta ciudad del país. En términos de planeación administrativa, la ciudad se divide en cinco localidades: Suroccidente, Suroriente, Norte-Centro Histórico, Metropolitana y Riomar (Figura 1) (Barranquilla, 2006). La ciudad, que limita por el oriente con el río Magdalena, principal arteria fluvial del país, se ubica en un entorno de humedales y ciénagas, hecho que da especificidad a sus problemas ambientales. Barranquilla depende del río para el desarrollo de muchas de sus actividades ya que es su única fuente hídrica. A su vez, el río es fuente de riesgos como inundaciones, deslizamientos y crecientes. Estas últimas, conocidas como arroyos, son corrientes de agua temporales que llenan algunas de sus calles durante los días de lluvia y generan pérdidas en las actividades económicas, daños a la infraestructura pública y, en no pocos casos, muertes por ahogamiento (Barranquilla Verde, 2017). En la localidad Suroriente, muchos de cuyos barrios se conformaron por invasión de predios baldíos, se encuentran una serie de canales laterales navegables denominados caños, que terminaron por convertirse en vertederos de aguas residuales y residuos sólidos y, con ello, en fuente de problemas de salud pública (Consejo Nacional de Política Económica y Social, 2005).

Según informes oficiales, el Î́ndice de Calidad Ambiental Urbana (ICAI) de la ciudad, observado por indicadores relativos a superficie de área verde, calidad del aire, calidad del agua, proporción de aguas protegidas, proporciones de población que participa en la gestión urbana, entre otros, es "muy bajo". Apenas un $0,13 \%$ de los residuos sólidos son aprovechados, solo 1 de cada 1000 habitantes participa en la gestión ambiental de la ciudad y cerca del $9 \%$ de su población se ubica en zonas de riesgo alto. En cuanto a la calidad del aire, si bien las mediciones mensuales realizadas por la autoridad ambiental de la ciudad indican que su nivel es "bueno", existen algunos eventos que la afectan temporalmente. Es el caso de los incendios intencionados que se producen en el Vía Parque Isla de Salamanca, un área protegida contigua a la ciudad y ubicada en el antiguo delta del río Magdalena. Estos incendios producen altas columnas de humo que cubren casi toda la ciudad y afectan principalmente a quienes residen cerca a la orilla del río (Barranquilla Verde, 2017).

En lo que respecta a la segregación espacial, Barranquilla exhibe patrones similares a los señalados para América Latina: las localidades ubicadas al norte (Norte-Centro Histórico y Riomar) tienen mejor habitabilidad, servicios e ingresos que las ubicadas en el centro sur (Suroccidente, Suroriente y Metropolitana). Esta distribución corresponde tanto a procesos históricos de ocupación como a las políticas urbanas que les fueron acompañando. La ciudad inició su poblamiento en el oriente durante el siglo XVI asociado al comercio entre las dos laderas del río y durante el siglo XIX esta área se fue consolidando producto de la invasión de baldíos y la urbanización de haciendas. Ya en el siglo XX, el auge portuario influyó en la puesta en marcha de un conjunto de proyectos de urbanización en el norte y centro, cuyos barrios, surgidos de modelos de planeación ordenados, albergaron a familias adineradas. Mientras tanto, el oriente y otros sectores del suroccidente crecieron de la mano de migrantes pobres provenientes del interior del país, que terminaron asentándose en lugares con poco acceso a servicios públicos (Barranquilla Verde, 2017).

Una aproximación a la territorialización desigual presente en Barranquilla es la del estrato. En Colombia, las condiciones de las viviendas se traducen en un sistema de estratificación para el cobro de los servicios públicos domiciliarios. Esta diferenciación tiene su origen en la década de los 60 del siglo pasado, cuando el Gobierno Nacional determinó que las empresas de servicios públicos deberían establecer un sistema de tarifas que respondiera a las particularidades socioeconómicas de los barrios (Yunda, 2019). A partir de entonces, los predios urbanos del país se clasificaron en 6 estratos que sintetizan información sobre las características de las viviendas y de su entorno. Así, el estrato 6 indica mejor habitabilidad y el 1 menor. 


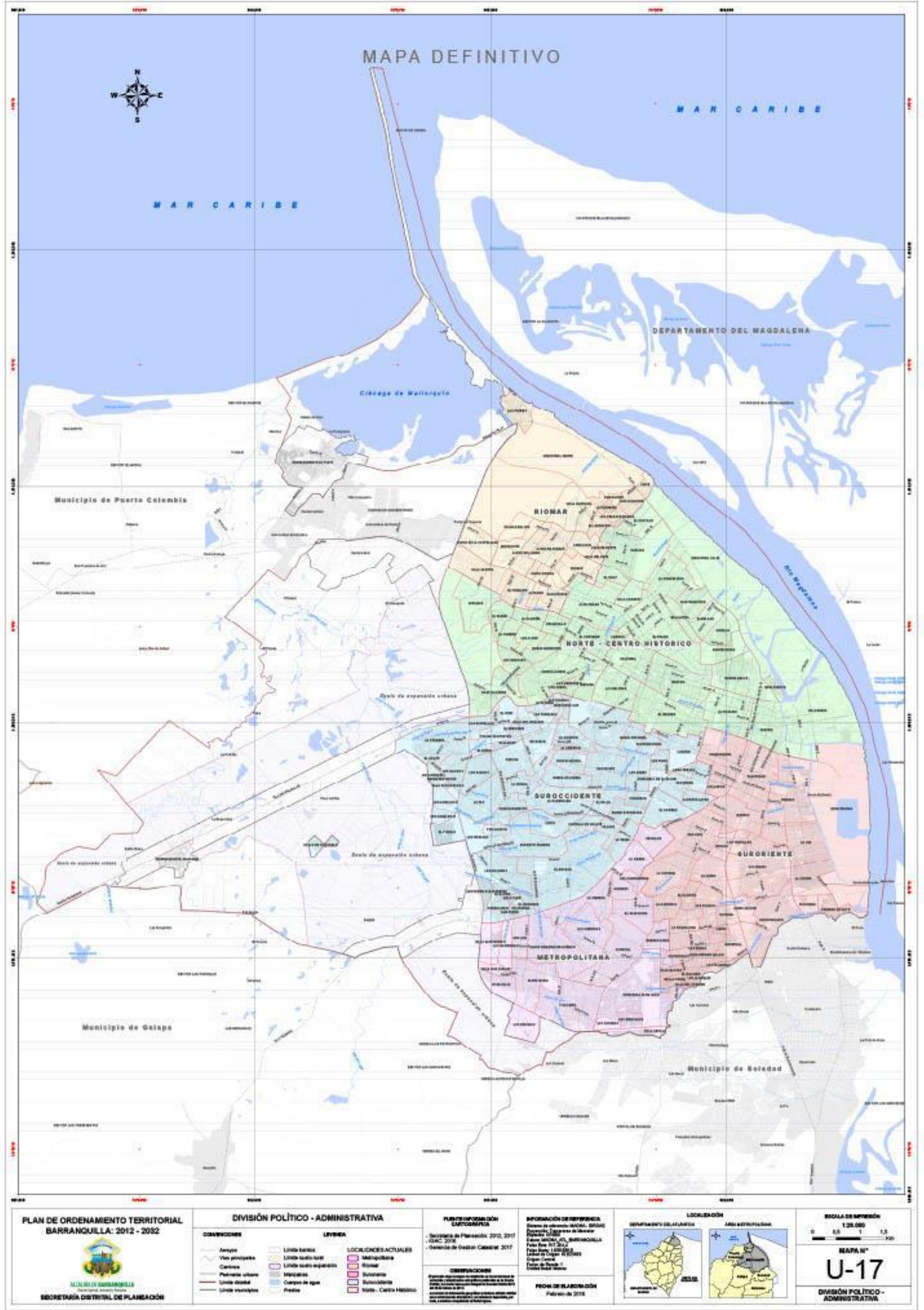

Figura 1 - Localidades de Barranquilla. Fuente: (Barranquilla, 2012). 
Aunque el estrato se toma de manera errónea como un indicador de ingreso de los hogares (Yunda, 2019), existen asociaciones entre estas variables. En Barranquilla, los ingresos de los hogares que habitan en viviendas de estrato 6, medidos por salarios mínimos de 2012, eran más de 6 veces los de aquellos en el 1, así mismo los gastos de los primeros triplicaban a los de los segundos (EDUBAR, 2012). Estas diferencias indican la segregación "acomodada" señalada por Rodríguez (2008) para otras ciudades de América Latina. Como se aprecia en el mapa 2 las viviendas de estratos altos tienen a concentrarse en el norte de la ciudad, mientras que las de estratos bajos se ubican en el sur. Los hogares de mayores ingresos se concentran también en el primer sector: mientras que Riomar y Norte-Centro Histórico tienen al 22,6\% y al 12,8\% de sus población bajo línea de pobreza, el 61,2\% de los habitantes de la localidad Suroccidente, el 49,6\% en Metropolitana y del 45,1\% de Suroriente viven en esa condición (Tabla 1) (Cepeda-Emiliani, 2013).

Tabla 1 - Datos sociodemográficos por localidad para la ciudad de Barranquilla

\begin{tabular}{llll}
\hline Localidad & $\begin{array}{l}\text { \% población por debajo de } \\
\text { Línea de pobreza }\end{array}$ & Línea de indigencia \\
\hline Suroccidente & 61,2 & 20,1 & 5,9 \\
Metropolitana & 49,6 & 15,1 & 5,6 \\
Suroriente & 45,1 & 18,5 & 5,8 \\
Riomar & 22,6 & 5,8 & 7,6 \\
Norte Centro Histórico & 12,8 & 4 & 8,5 \\
\hline
\end{tabular}

Fuente: Elaboración propia con base en Cepeda Emiliani (2013, pp. 10-11).

Un hecho relacionado con la espacialización de la riqueza y del bienestar en Barranquilla es que el estrato ha pasado a ser un indicador de estatus social y fuente de exclusión. Esto es similar a lo sucedido en el Gran Mendoza, en Ciudad de México, en Bogotá y en otras ciudades latinoamericanas donde la segregación espacial opera como fuente de estereotipos de discriminación, pero también de identidad (Rodríguez, 2008; Saraví, 2008; Tosoni, 2011). Nos preguntamos entonces si este patrón guarda relación con la satisfacción de sus habitantes y con sus comportamientos proambientales. Para responder a esta pregunta en lo que sigue presentamos los resultados del análisis de la Encuesta de Percepción Ciudadana 2017 aplicada por Barranquilla Cómo Vamos.

\section{Metodología y resultados}

La Encuesta de Percepción Ciudadana es una encuesta anual que pregunta por las percepciones de los ciudadanos en cuanto a cuatro conjuntos de variables: clima de opinión, activos de las personas, atributos urbanos y buen gobierno y ciudadanía. Para responder a la pregunta sobre la relación entre segregación, satisfacción con el entorno y comportamientos proambientales, realizamos dos ejercicios estadísticos ( $n=1246)$ : análisis de asociación entre las variables de localidad y estrato de las viviendas, de un lado y entre la satisfacción con los equipamientos urbanos y la vivienda, de otro. Además, un agrupamiento jerárquico (hierarchical clustering) para indagar por la existencia de conglomerados de comportamientos. Para ello, utilizamos el paquete FactoMineR (Lê et al., 2008) en el software estadístico R. Las variables y categorías de respuesta se recogen en la Tabla 2 . 


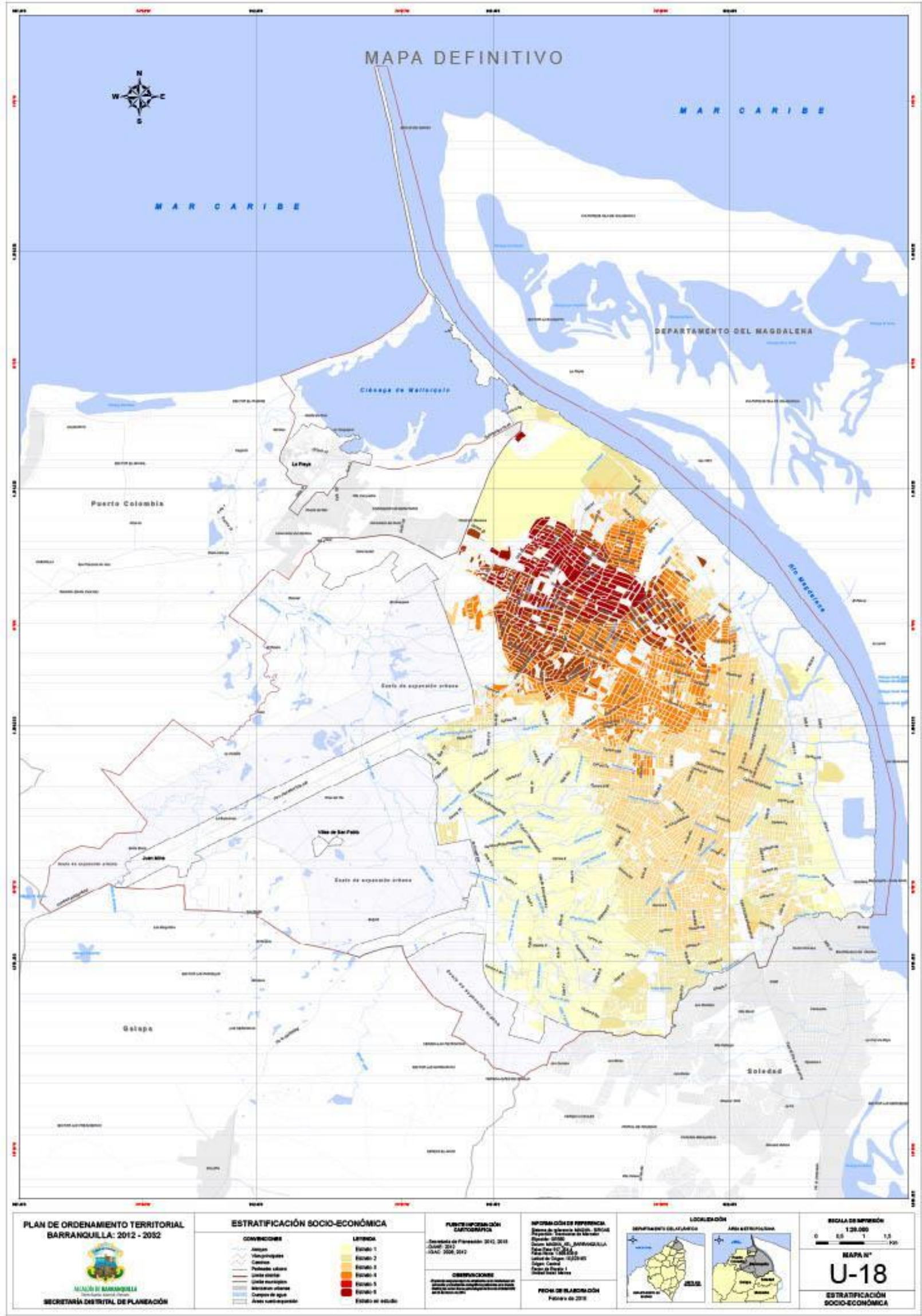

Figura 2 - Estratificación socioeconómica Barranquilla. Colores más cercanos al rojo representan estratos altos, más cercanos al amarillo representan a los bajos. Fuente: Barranquilla (2012). 
Tabla 2 - Variables y categorías de respuesta incluidas en el análisis

\begin{tabular}{|c|c|}
\hline Variable & Categorías \\
\hline \multirow{2}{*}{ Género } & Hombre \\
\hline & Mujer \\
\hline \multirow[t]{5}{*}{ Edad } & 18-25 años \\
\hline & 26-35 años \\
\hline & 36-45 años \\
\hline & 46-55 años \\
\hline & Más de 55 años \\
\hline \multirow[t]{5}{*}{ Localidad } & Riomar \\
\hline & Norte Centro Histórico \\
\hline & Suroriental \\
\hline & Metropolitana \\
\hline & Suroccidente \\
\hline \multirow[t]{6}{*}{ Estrato } & Estratol \\
\hline & Estrato2 \\
\hline & Estrato3 \\
\hline & Estrato4 \\
\hline & Estrato5 \\
\hline & Estrato6 \\
\hline \multirow[t]{4}{*}{$\dot{\imath}$ Usted vive en una vivienda? } & Propia \\
\hline & Arrendada \\
\hline & En usufructo \\
\hline & Ocupante de hecho \\
\hline ¿Qué tan satisfecho se siente con la vivienda que habita? & Escala Likert de 5 \\
\hline ¿Qué tan satisfecho(a) está usted en general con el barrio donde vive? & Escala Likert de 5 \\
\hline ¿Qué tan satisfecho(a) está usted en general con el alumbrado público de su barrio? & Escala Likert de 5 \\
\hline $\begin{array}{l}\text { ¿Qué tan satisfecho(a) está usted en general con los parques y zonas verdes públicas } \\
\text { de su barrio? }\end{array}$ & Escala Likert de 5 \\
\hline $\begin{array}{l}\text { ¿Qué tan satisfecho(a) está usted en general con los andenes y separadores de las } \\
\text { calles en su barrio? }\end{array}$ & Escala Likert de 5 \\
\hline $\begin{array}{l}\text { ¿Qué tan satisfecho(a) está usted en general con el estado de las vías en general de } \\
\text { Barranquilla? }\end{array}$ & Escala Likert de 5 \\
\hline Desconectan los aparatos eléctricos o electrónicos cuando no están en uso & Sí/No \\
\hline Llevan bolsas no plásticas al supermercado & Sí/No \\
\hline Usan con poca frecuencia los aparatos de calefacción o ventilación & Sí/No \\
\hline Ahorran agua & Sí/No \\
\hline Reciclan & Sí/No \\
\hline Cuidan las zonas verdes & Sí/No \\
\hline No arrojan basuras a las calles, quebradas o ríos & Sí/No \\
\hline
\end{tabular}

Fuente: Elaboración propia.

La primera revisión consistió en cruzar las variables de localidad y estrato de las viviendas con las relativas a la satisfacción con la vivienda y con el barrio, sus equipamientos del barrio, y con las viviendas, con el fin de determinar si había entre ellas alguna relación estadísticamente significativa validada con pruebas de chi cuadrado. Para robustecer el poder de la prueba, las variables de satisfacción, recogidas en escala de Likert de 5, se agruparon en niveles de 1 a 3 como "insatisfecho" y de 4 a 5 como "satisfecho". La variable estrato fue agrupada en tres categorías: bajo (estratos 1 y 2), medio ( 3 y 4), alto (5 y 6 ).

Pese al hecho de que las localidades tienen distintas condiciones de habitabilidad, la satisfacción con el barrio y con la vivienda es superior al 79\% en todos los casos. No obstante, los resultados muestran que hay relación estadísticamente significativa entre las variables analizadas: las localidades más acomodadas tienen mayores niveles de satisfacción y las de menores ingresos mayores niveles de insatisfacción. De manera específica, la localidad Norte Centro Histórico, tiene niveles de satisfacción por encima de la media en casi todos los aspectos, salvo en las vías. Por su parte, la localidad Suroccidente, tiene satisfacciones por 
debajo para todos los aspectos con excepción del alumbrado público. Los resultados para la insatisfacción, la percepción opuesta, son similares a los anteriores, aunque aquí hay más diferencias: la localidad Norte Centro Histórico tiene niveles por debajo de la media para todos los equipamientos, menos para vías, y niveles superiores para la vivienda. Por su parte, la localidad Suroccidente tiene niveles de insatisfacción por encima de la media para todos los equipamientos, menos para alumbrado, y niveles menores para la vivienda. Además, los habitantes de la localidad Metropolitana tienen niveles de insatisfacción superiores para alumbrado y parques y zonas verdes, e inferiores para vías, percepción última que comparte con Suroriente (Barrio: chi2 $=18.115$, gdl. $=4, \mathrm{p}=0.0012$; alumbrado: $\operatorname{chi} 2=16.933$, gdl. $=4, \mathrm{p}=0.0020$; parques y zonas verdes: chi2 $=73.43$, gdl. $=4, p<0,001$; andenes y separadores: chi2 $=21.577$, gdl. $=4, p=0.00024$; vías: $\operatorname{chi} 2=16.47$, gdl. $=4, \mathrm{p}=0.0024$; vivienda: chi2 $=13.905$, gdl. $=4, \mathrm{p}=0.0076$ ) (Tabla 3).

Tabla 3 - Localidad y satisfacción con los equipamientos del barrio y con la vivienda

\begin{tabular}{|c|c|c|c|c|c|c|c|}
\hline & $\begin{array}{l}\text { Localidad } \\
(\%, n=1246)\end{array}$ & $\begin{array}{l}\text { Barri } \\
0\end{array}$ & $\begin{array}{l}\text { Alumbrad } \\
\text { ○ }\end{array}$ & $\begin{array}{l}\text { Parques y zonas } \\
\text { verdes }\end{array}$ & $\begin{array}{l}\text { Andenes y } \\
\text { separadores }\end{array}$ & Vías & $\begin{array}{l}\text { Viviend } \\
\text { a }\end{array}$ \\
\hline \multirow{6}{*}{ 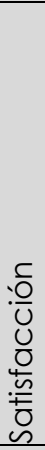 } & Riomar & 88.6 & 66.7 & $71,4^{*}$ & 62.9 & 48.6 & 93.3 \\
\hline & $\begin{array}{l}\text { Norte Centro } \\
\text { Histórico }\end{array}$ & $89,7^{*}$ & $73,2^{*}$ & $76,1^{*}$ & $71,8^{*}$ & 52.1 & $94,4^{*}$ \\
\hline & Suroriente & 82.2 & 65.9 & 57.2 & 59.1 & $\begin{array}{l}61,2 \\
*\end{array}$ & 90.3 \\
\hline & Metropolitana & 81.2 & 56.4 & $41,0^{*}$ & 58.8 & $\begin{array}{l}62,0 \\
*\end{array}$ & 93.6 \\
\hline & Suroccidente & $76,9^{*}$ & 60.0 & $49,0^{*}$ & $52,3^{*}$ & $\begin{array}{l}49,4 \\
*\end{array}$ & $86,5^{*}$ \\
\hline & Total & 82.3 & 63.7 & 56.3 & 59.5 & 55.3 & 90.8 \\
\hline \multirow{6}{*}{ 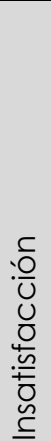 } & Riomar & 11,4 & 33,3 & $28,6^{*}$ & 37,1 & 51,4 & 6,7 \\
\hline & $\begin{array}{l}\text { Norte Centro } \\
\text { Histórico }\end{array}$ & $10,3^{*}$ & $26,8^{*}$ & $23,9^{*}$ & $28,2^{*}$ & 47,9 & $5,6^{*}$ \\
\hline & Suroriente & 17,8 & 34,1 & 42,8 & 40,9 & $\begin{array}{l}38,8 \\
*\end{array}$ & 9,7 \\
\hline & Metropolitana & 18,8 & $43,6^{*}$ & $59,0^{*}$ & 41,2 & $\begin{array}{l}38,0 \\
*\end{array}$ & 6,4 \\
\hline & Suroccidente & $23,1^{*}$ & 40,0 & $51,0^{*}$ & $47,7^{*}$ & $\begin{array}{l}50,6 \\
*\end{array}$ & $13,5^{*}$ \\
\hline & Total & 17,7 & 36,3 & 43,7 & 40,3 & 44,7 & 9,2 \\
\hline
\end{tabular}

Nota: * Residuos estandarizados absolutos mayores a 1,96. Fuente: Elaboración propia.

Los resultados respecto al estrato de las viviendas son compatibles con los de la localidad, pero en este caso las diferencias son más significativas. Las personas que viven en viviendas de estrato 1 y 2 tienen niveles de satisfacción e insatisfacción con el barrio por encima de la media. No hay diferencias en cuanto a la satisfacción o insatisfacción con las vías. En cambio, si se presentan distancias en cuanto a los demás equipamientos. En particular, las personas que viven en viviendas de estrato 1 y 2 tienen satisfacciones por debajo e insatisfacciones por encima de la media para todos los demás elementos, situación opuesta a quienes viven en viviendas de estratos 5 y 6. Quienes habitan en viviendas de estratos 3 y 4 se parece en mucho a este último y se distingue, también en mucho, del primero. No obstante, comparte con éste su insatisfacción con el alumbrado público (Barrio: chi2 = 31.331, gdl = 2, p<0,001; alumbrado: chi2 = 34.045, gdl. $=2$, p<0,001; parques y zonas verdes: chi2 $=96.64$, gdl. $=2, \mathrm{p}<0,001$; andenes y separadores: chi $2=$ 31,097, g.d.l. $=2, \mathrm{p}<0,001$; vías: chi2 $=11.636$, gdl. $=2, \mathrm{p}<0,001$; vivienda: chi $2=11.636$, gdl. $=2, \mathrm{p}<0,001$ ) 
Segregación espacial, satisfacción con el barrio y comportamientos responsables con el medio ambiente en una ciudad latinoamericana

Tabla 4 - Estrato de las viviendas y satisfacción con los equipamientos del barrio y con la vivienda.

\begin{tabular}{|c|c|c|c|c|c|c|c|}
\hline & $\begin{array}{l}\text { Estrato } \\
(\%, n=1246)\end{array}$ & Barrio & Alumbrado & Parques y zonas verdes & Andenes y separadores & Vías & Vivienda \\
\hline \multirow{4}{*}{ 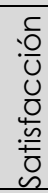 } & 1 y 2 & $76,8^{*}$ & $56,9^{*}$ & $43,2^{*}$ & $52,4^{*}$ & 44,7 & $88,1^{*}$ \\
\hline & 3 y 4 & $87,1^{*}$ & $68,8^{*}$ & $68,4^{*}$ & $65,5^{*}$ & 44,7 & $93,1^{*}$ \\
\hline & 5 y 6 & $93,2^{*}$ & $80,5^{*}$ & $78,2^{*}$ & $73,7^{*}$ & 44,7 & $95,5^{*}$ \\
\hline & Total & 82,4 & 63,9 & 56,3 & 59,6 & 44,7 & 90,8 \\
\hline \multirow{4}{*}{ 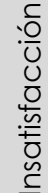 } & 1 y 2 & $23,2^{*}$ & $43,1^{*}$ & $56,8^{*}$ & $47,6^{*}$ & 55,3 & $11,9^{*}$ \\
\hline & 3 y 4 & $12,9 *$ & $31,2^{*}$ & $31,6^{*}$ & $34,5^{*}$ & 55,3 & $6,9^{*}$ \\
\hline & 5 y 6 & $6,8^{*}$ & $19,5^{*}$ & $21,8^{*}$ & $26,3^{*}$ & 55,3 & $4,5^{*}$ \\
\hline & Total & 17,6 & 36,1 & 43,7 & 40,4 & 55,3 & 9,2 \\
\hline
\end{tabular}

Nota: * Residuos estandarizados absolutos mayores a 1,96. Fuente: Elaboración propia.

El agrupamiento jerárquico (hierarchical clustering) es una técnica estadística de clasificación de datos que busca situar los casos en grupos homogéneos que no son conocidos, pero que son sugeridos por los datos. De este modo, individuos similares son asignados a un mismo grupo e individuos distintos son asignados a grupos distintos (Pérez, 2004). Un primer ejercicio nos permitió identificar tres grupos que nombramos, según sus respuestas a las preguntas por la satisfacción con los equipamientos del barrio y con sus viviendas, como Críticos ( $\mathrm{n}=847,68,0 \%)$, Satisfechos $(\mathrm{n}=376,30,2 \%)$, y un grupo residual $(\mathrm{n}=23,1,8 \%)$ que se distingue por sus bajas tasas de respuesta. Para efectos de este análisis tomamos los dos primeros. El grupo de los Críticos reúne a personas que se encuentran en el rango que va de algo de satisfacción a mucha insatisfacción con el barrio, los parques y zonas verdes, el alumbrado público, los separadores, las vías y su vivienda. Además, recoge a personas de 36 a 45 años, habitantes de la localidad Metropolitana, con vivienda propia y de estrato 1 (Tabla 5).

Tabla 5 - Grupo Críticos $(n=847,68,0 \%)$

\begin{tabular}{|c|c|c|c|c|c|}
\hline Categoría & Cat\% & Clúster\% & Global\% & p.value & v.test \\
\hline Algo satisfecho barrio & 93,9 & 54,8 & 39,6 & 0,000 & 17,1 \\
\hline Algo satisfecho parques zonas verdes & 95,3 & 40,3 & 28,7 & 0,000 & 14,5 \\
\hline Algo satisfecho alumbrado & 90,3 & 48,3 & 36,4 & 0,000 & 13,5 \\
\hline Algo satisfecho andenes y separadores & 90,4 & 45,8 & 34,4 & 0,000 & 13,1 \\
\hline Algo satisfecho vivienda & 89,9 & 41,9 & 31,7 & 0,000 & 12,0 \\
\hline Algo satisfecho vías & 85,7 & 44,6 & 35,4 & 0,000 & 10,3 \\
\hline Ni satisfecho ni insatisfecho alumbrado & 91,6 & 24,3 & 18,1 & 0,000 & 9,1 \\
\hline Ni satisfecho ni insatisfecho parques zonas verdes & 94,4 & 19,7 & 14,2 & 0,000 & 9,1 \\
\hline Ni satisfecho ni insatisfecho andenes y separadores & 88,9 & 26,6 & 20,3 & 0,000 & 8,6 \\
\hline Ni satisfecho ni insatisfecho barrio & 94,5 & 14,2 & 10,2 & 0,000 & 7,6 \\
\hline Algo insatisfecho andenes y separadores & 90,8 & 13,9 & 10,4 & 0,000 & 6,4 \\
\hline Ni satisfecho ni insatisfecho vías & 81,0 & 33,3 & 27,9 & 0,000 & 6,3 \\
\hline Algo insatisfecho alumbrado & 89,3 & 11,8 & 9,0 & 0,000 & 5,5 \\
\hline Algo insatisfecho vías & 85,3 & 11,0 & 8,7 & 0,000 & 4,3 \\
\hline Algo insatisfecho parques zonas verdes & 84,6 & 10,4 & 8,3 & 0,000 & 4,0 \\
\hline Muy insatisfecho parques zonas verdes & 77,6 & 23,3 & 20,4 & 0,000 & 3,7 \\
\hline Reciclan & 76,0 & 28,8 & 25,8 & 0,000 & 3,6 \\
\hline Algo insatisfecho barrio & 88,2 & 5,3 & 4,1 & 0,001 & 3,4 \\
\hline Muy insatisfecho andenes y separadores & 80,9 & 10,5 & 8,8 & 0,002 & 3,1 \\
\hline Vivienda propia & 70,3 & 74,0 & 71,6 & 0,006 & 2,8 \\
\hline Metropolitana & 74,8 & 20,7 & 18,8 & 0,012 & 2,5 \\
\hline Ni satisfecho ni insatisfecho vivienda & 80,6 & 6,4 & 5,4 & 0,020 & 2,3 \\
\hline estrato 1 & 73,0 & 27,4 & 25,5 & 0,027 & 2,2 \\
\hline De 36 a 45 años & 74,7 & 16,1 & 14,6 & 0,033 & 2,1 \\
\hline
\end{tabular}

Nota: Categoría: categorías de respuesta a las preguntas sociodemográficas y barrio y vivienda recogidas en el grupo; Cat\%: proporción de la categoría en el grupo; Clúster\%: proporción del grupo en la categoría; Global\%: proporción de la categoría 
dentro del conjunto de categorías de respuesta; p.valor: valor p; v.test; valores test, índice de ordenamiento que identifica las categorías de respuesta que más identifican al grupo, a mayor valor mayor identificación. Se excluyen valores negativos por cuanto no identifican al grupo. Fuente: Elaboración propia.

El segundo grupo, los Satisfechos, se identifica por estar muy satisfecho con los andenes y separadores, el alumbrado público, los parques y zonas verdes, las vías y el barrio. Además, agrupa a encuestados que viven la localidad Riomar, en viviendas arrendadas y estratos 6 y 5 (Tabla 6).

Tabla 6 - Grupo Satisfechos ( $n=376,30,2 \%)$

\begin{tabular}{|c|c|c|c|c|c|}
\hline Categoría & Cat\% & Cluster\% & Global\% & p.value & v.test \\
\hline Muy satisfecho andenes y separadores & 91,6 & 72,9 & 24,0 & 0,000 & 26,5 \\
\hline Muy satisfecho parques zonas verdes & 83,6 & 73,4 & 26,5 & 0,000 & 24,5 \\
\hline Muy satisfecho alumbrado & 83,7 & 72,6 & 26,2 & 0,000 & 24,3 \\
\hline Muy satisfecho barrio & 63,1 & 85,9 & 41,1 & 0,000 & 21,7 \\
\hline Muy satisfecho vías & 87,2 & 54,5 & 18,9 & 0,000 & 20,6 \\
\hline Muy satisfecho vivienda & 43,7 & 82,7 & 57,1 & 0,000 & 12,4 \\
\hline Riomar & 43,2 & 12,8 & 8,9 & 0,002 & 3,0 \\
\hline Vivienda arrendada & 37,4 & 24,5 & 19,7 & 0,007 & 2,7 \\
\hline estrato 6 & 53,1 & 4,5 & 2,6 & 0,007 & 2,7 \\
\hline estrato 5 & 42,2 & 11,4 & 8,2 & 0,008 & 2,7 \\
\hline
\end{tabular}

Nota: Categoría: categorías de respuesta a las preguntas sociodemográficas y barrio y vivienda recogidas en el grupo; Cat\%: proporción de la categoría en el grupo; Clúster\%: proporción del grupo en la categoría; Global\%: proporción de la categoría dentro del conjunto de categorías de respuesta; p.valor: valor p; v.test; valores test, índice de ordenamiento que identifica las categorías de respuesta que más identifican al grupo, a mayor valor mayor identificación. Se excluyen valores negativos por cuanto no identifican al grupo. Fuente: Elaboración propia.

Con los grupos identificados incorporamos las respuestas a las preguntas relativas a los comportamientos responsables con el medio ambiente. El ejercicio mostró diferencias adicionales, que denominamos ahora como Críticos-Ahorradores y Satisfechos-Derrochadores. Además de las características ya señaladas, el primero recoge a personas que desconectan los aparatos eléctricos cuando no están en uso, ahorran agua y usan con poca frecuencia los aparatos de calefacción o ventilación. Sin embargo, no manifiestan llevar bolsas no plásticas al supermercado o no arrojar basuras a las calles, quebradas o ríos (Tabla 7). Los segundos se asocian con comportamientos como llevar bolsas plásticas al supermercado, pero no manifiestan desconectar los aparatos eléctricos cuando no están en uso, usar con poca frecuencia aparatos de calefacción o ventilación, ahorrar agua, reciclar o no arrojar basuras a las calles, quebradas o ríos (Tabla 8).

Tabla 7 - Grupo Críticos-Ahorradores ( $\mathrm{n}=847,68,0 \%$ )

\begin{tabular}{|c|c|c|c|c|c|}
\hline Categoría & Cat\% & Clúster\% & Global\% & p.value & v.test \\
\hline Arrojan basuras a las calles, quebradas o ríos & 77,9 & 40,3 & 35,2 & 0,000 & 5,6 \\
\hline Ahorran agua & 72,9 & 67,7 & 63,1 & 0,000 & 4,8 \\
\hline \multicolumn{6}{|l|}{ Desconectan los aparatos eléctricos o electrónicos cuando no están en } \\
\hline USO & 74,6 & 51,7 & 47,1 & 0,000 & 4,8 \\
\hline Usan con poca frecuencia los aparatos de calefacción o ventilación & 83,5 & 9,6 & 7,8 & 0,000 & 3,6 \\
\hline No llevan bolsas no plásticas al supermercado & 69,9 & 82,6 & 80,4 & 0,004 & 2,8 \\
\hline
\end{tabular}

Nota: Categoría: categorías de respuesta a las preguntas sociodemográficas y barrio y vivienda recogidas en el grupo; Cat\%: proporción de la categoría en el grupo; Clúster\%: proporción del grupo en la categoría; Global\%: proporción de la categoría dentro del conjunto de categorías de respuesta; p.valor: valor p; v.test; valores test, índice de ordenamiento que identifica las categorías de respuesta que más identifican al grupo, a mayor valor mayor identificación. Se excluyen valores negativos por cuanto no identifican al grupo. Fuente: Elaboración propia. 
Segregación espacial, satisfacción con el barrio y comportamientos responsables con el medio ambiente en una ciudad latinoamericana

Tabla 8 - Grupo Satisfechos-Derrochadores ( $n=376,30,2 \%$ )

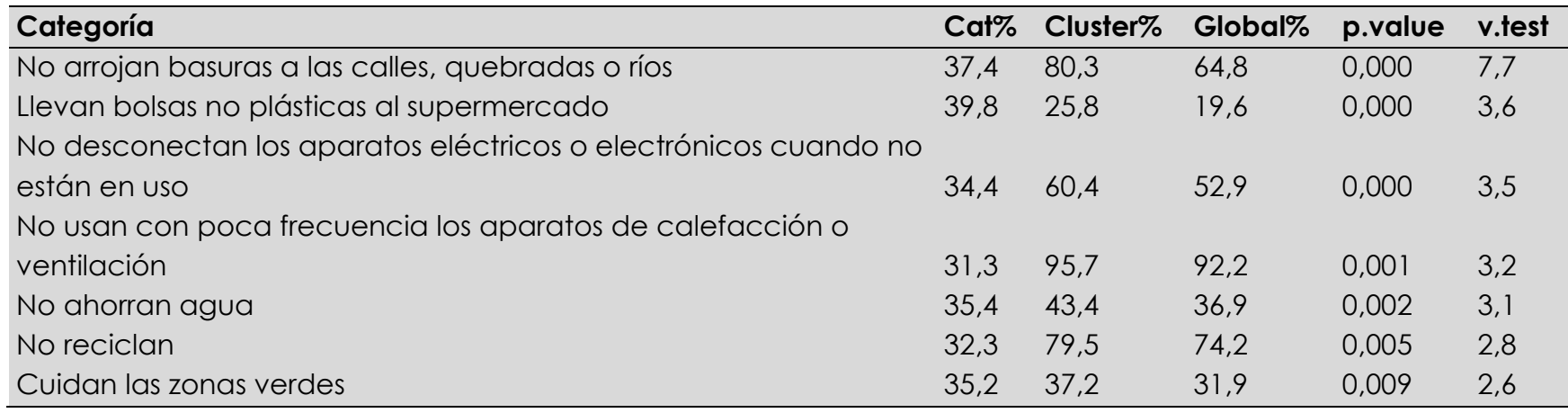

Nota: Categoría: categorías de respuesta a las preguntas sociodemográficas y barrio y vivienda recogidas en el grupo; Cat\%: proporción de la categoría en el grupo; Clúster\%: proporción del grupo en la categoría; Global\%: proporción de la categoría dentro del conjunto de categorías de respuesta; p.valor: valor p; v.test; valores test, índice de ordenamiento que identifica las categorías de respuesta que más identifican al grupo, a mayor valor mayor identificación. Se excluyen valores negativos por cuanto no identifican al grupo. Fuente: Elaboración propia.

\section{Discusión y conclusiones}

En este artículo presentamos los resultados de un análisis de asociación y de agrupamiento jerárquico, para un conjunto de respuestas relativas a la localidad, el estrato de las viviendas, la satisfacción con el barrio, la vivienda y los equipamientos urbanos, de un lado y las conductas responsables con el medio ambiente, del otro, contenidas en la Encuesta de Percepción Ciudadana de Barranquilla Cómo Vamos 2017. En particular, indagamos por la relación existente entre segregación espacial, satisfacción con el entorno, como indicador del efecto barrio, y conductas responsables con el medio ambiente.

En Barranquilla, las localidades y los estratos de las viviendas comparten un patrón de distribución espacial en el que las localidades ubicadas en el norte (Río Mar y Norte-Centro Histórico) concentran viviendas de estratos altos y las localidades ubicadas en el sur (Suroccidente, Suroriente y Metropolitana) concentran viviendas de estratos bajos (Figuras 1 y 2). El análisis de asociación muestra diferencias para la satisfacción con el entorno influidas por ambos aspectos. La localidad Norte Centro Histórico, la de mejores ingresos tiene niveles de satisfacción estadísticamente significativos por encima de la media para la satisfacción con el barrio, alumbrado, parques y zonas verdes, andenes y separadores y vivienda. Por su parte, la localidad Suroccidente, que tiene la mayor proporción de habitantes por debajo de la línea de pobreza de toda la ciudad, tiene satisfacciones estadísticamente significativas por debajo de la media, para satisfacción con el barrio, parques y zonas verdes, andenes y separadores, vías y vivienda. En cuanto a los estratos de las viviendas, encontramos que quienes habitan en estratos bajos tienen niveles de satisfacción por debajo de la media para todos los aspectos preguntados, con excepción de las vías. Por su parte, quienes viven en viviendas de estratos altos tienen niveles de satisfacción por encima de la media para todos los aspectos preguntados, con la misma excepción.

El análisis de agrupamiento jerárquico (hierarchical clustering) muestra dos grupos diferenciados en cuanto a la satisfacción con el entorno. El primero, denominado como Críticos ( $\mathrm{n}=847,68,0 \%)$, recoge personas que viven en la localidad Metropolitana, una de las de menores ingresos, ubicada en el sur de la ciudad y en estrato 1. Estas personas tienen rangos de satisfacción que van de algo de satisfacción a mucha insatisfacción con los equipamientos del barrio, lo que es consistente con el análisis de asociación. El segundo grupo, los Satisfechos $(n=376,30,2 \%)$, recoge personas que viven en estratos 5 y 6 , el de mayores ingresos y mejores viviendas y en la localidad Riomar, en el norte de la ciudad. Según las preguntas analizadas, este grupo se encuentra muy satisfecho con su barrio y sus equipamientos.

En cuanto a los comportamientos responsables con el medio ambiente, las personas Críticas son también Ahorradoras: desconectan aparatos eléctricos, usan con poca frecuencia apartados de calefacción o ventilación, ahorran agua y reciclan. No obstante, no manifiestan no arrojar basuras a las calles, quebradas 
o ríos. Las personas Satisfechas, por su parte, se asocian a comportamientos que denominamos Derrochadores: no afirman desconectar aparatos eléctricos, usar con poca frecuencia aparatos de calefacción o ventilación, ahorrar agua o reciclar. Sin embargo, a diferencia del primer grupo, manifiestan no arrojar basuras a los espacios públicos y llevan bolsas plásticas al supermercado.

Los hallazgos son consistentes con la existencia de un efecto barrio asociado a la satisfacción el entorno urbano, que se vincula con los "patrones de calidad residencial" (Amérigo \& Aragonés, 1997) y con los comportamientos proambientales. No obstante, el análisis sugiere matices que merecen ser explorados. En primer lugar, si bien el grupo de Críticos-Ahorradores manifiesta en sus respuestas realizar acciones responsables con el medio ambiente, éstas suceden en los espacios privados o domésticos. Esto es opuesto a lo que sucede con las personas Satisfechas-Derrochadoras, cuyas acciones responsables suceden en espacios públicos. Las investigaciones sobre estos comportamientos distinguen entre los que tienen lugar en la esfera pública y privada con base en la distinción activismo/no activismo, el primero asociado con la participación en organizaciones proambientales (Blankenberg \& Alhusen, 2019; Stern et al., 1999). No obstante, lo que este análisis sugiere es una distinción al interior de los comportamientos no activistas: las personas que viven en viviendas de estratos bajos se encuentran menos satisfechas con su entorno y circunscriben sus comportamientos proambientales al ámbito privado (el hogar). Por su parte, las personas que viven en viviendas de estratos altos, tienen niveles de satisfacción mayores y orientan sus comportamientos al ámbito público.

En segundo lugar, un hallazgo que merece ser notado es que los Críticos-Ahorradores agrupan a personas propietarias de sus viviendas, mientras que el grupo de los Satisfechos-Derrochadores a personas arrendatarias. En este análisis estos tipos se asocian a la insatisfacción/satisfacción con el entorno de forma respectiva, hecho contrario a la idea de que los propietarios se encuentran más satisfechos con su barrio dado que tienen más opciones dentro del mercado inmobiliario (Permentier et al., 2010, p. 980), lo que sugiere relaciones más complejas entre segregación, satisfacción y comportamientos. Es posible entonces la existencia de un "efecto propietario" asociado al efecto barrio en el que la relación de propiedad orienta los comportamientos hacia ámbitos públicos o privados según el caso. Para evaluar esta relación será necesario distinguir en los análisis estos tipos de comportamientos, así como incorporar en ellos la relación jurídica de las personas con sus viviendas: los dos campos están por explorar.

\section{Declaración de disponibilidad de datos}

El conjunto de datos que respalda los resultados de este artículo está disponible en SciELO DATA y se puede acceder a él en https://doi.org/10.48331/scielodata.06HBOP

\section{Referencias}

Amérigo, M., \& Aragonés, J. I. (1997). A Theoretical and Methogological Approach to the Study of Residential Satisfaction. Journal of Environmental Psychology, 17(1), 47-57. https://doi.org/10.1006/JEVP.1996.0038

Bai, X., McPhearson, T., Cleugh, H., Nagendra, H., Tong, X., Zhu, T., \& Zhu, Y.-G. (2017). Linking Urbanization and the Environment: Conceptual and Empirical Advances. Annual Review of Environment and Resources, 42(1), 215-240. https://doi.org/10.1146/annurev-environ-102016-061128

Barranquilla. (2006). Acuerdo por el cual se determinan las localidades de la ciudad de Barranquilla.

Barranquilla. (2012). Plan de Ordenamiento Territorial 2012-2032. Recuperado en 29 de junio de 2021 de https://goo.gl/3QEaYp

Barranquilla Verde. (2017). Plan de gestión ambiental distrital de Barranquilla 2017 - 2027. Recuperado en 29 de junio de 2021 de http://barranquillaverde.gov.co/planeacion-gestion-y-control/plan-de-gestion-ambiental-distrital-debarranquilla-2017-2027 
Bittencourt, T. A., \& Faria, J. R. V. de. (2021). Distribuição de investimentos públicos, infraestrutura urbana e desigualdade socioespacial em Curitiba. urbe. Revista Brasileira de Gestão Urbana, 13. https://doi.org/10.1590/2175-

3369.013.e20190300

Blankenberg, A.-K., \& Alhusen, H. (2019). On the Determinants of Pro-Environmental Behavior: A Literature Review and Guide for Empirical Economist (No. 350). Recuperado en 29 de junio de 2021 de http://wwwuser.gwdg.de/ cege/Diskussionspapiere/DP350.pdf

Borges, Â., \& Carvalho, I. (2017). Revisiter les effets de lieu: Ségrégation et accès au marché du travail dans une métropole Brésilienne. Caderno CRH, 30(79), 121-135. https://doi.org/10.1590/S0103-49792017000100008

Bourdieu, P. (2007). Efectos de lugar. In Pierre Bourdieu (Ed.), La miseria del mundo (pp. 119-124). Buenos Aires: Fondo de Cultura Económica.

Brown, Z. (2014). Greening Household Behavior: Cross-domain Comparisons in Environmental Attitudes and Behaviours Using Spatial Effectis (No. 68). https://doi.org/10.1787/5jxrclsj8z7b-en\%0A

Cepeda-Emiliani, L. (2013). Los sures de Barranquilla : la distribución espacial de la pobreza. In L. Cepeda-Emiliani, $L a$ economía de Barranquilla a comienzos del siglo XXI. Banco de la República de Colombia. Recuperado en 29 de junio de 2021 de http://repositorio.banrep.gov.co/handle/20.500.12134/6659

Consejo Nacional de Política Económica. (2005). Importancia estratégica del plan de saneamiento de los caños de la cuenca oriental de la ciudad de Barranquilla. Recuperado en 29 de junio de 2021 de https://colaboracion.dnp.gov.co/CDT/Conpes/Económicos/3351.pdf

Crociata, A., Agovino, M., \& Sacco, P. L. L. (2016). Neighborhood effects and pro-environmental behavior: The case of Italian separate waste collection. Journal of Cleaner Production, 135, 80-89. https://doi.org/10.1016/J.JCLEPR0.2016.06.083

Delamaza, G., \& Thayer, L. E. (2016). Percepciones políticas y prácticas de participación como instrumento para la gobernanza de los territorios. EURE (Santiago), 42(127), 137-158. https://doi.org/10.4067/S0250-71612016000300006

Economic Commission for Latin America and the Caribbean - ECLCAC. (2016). Challenges, dilemmas and commitments of a common urban agenda Executive summary. Recuperado en 29 de junio de 2021 de https://repositorio.cepal.org//handle/11362/40657

Edubar. (2012). Estudio socioeconómico y determinación de la capacidad del sector residencial del distrito de Barranquilla para la contribución del valorización por beneficio general. Recuperado en 29 de junio de 2021 de https://goo.gl/ky9w5t

Ellen, I. G., \& Turner, M. A. (1997). Does Neighborhood Matter? Assessing Recent Evidence. Housing Policy Debate, 8(4), 833-866. https://doi.org/10.1080/10511482.1997.9521280

Galster, G. C., \& Killen, S. P. (1995). The Geography of Metropolitan Opportunity: A Reconnaissance and Conceptual Framework. Housing Policy Debate, 6(1), 7-43. https://doi.org/10.1080/10511482.1995.9521180

Gilbert, A., \& Ferguson, J. (1994). 5. Housing Strategies. In The Latin American City (pp. 79-102). https://doi.org/10.3362/9781909013841.005

Kearns, A., \& Parkinson, M. (2001). The significance of neighbourhood. Urban Studies, 38(12), 2103-2110. https://doi.org/10.1080/00420980120087063

Lê, S., Josse, J., \& Husson, F. (2008). FactoMineR : An R Package for Multivariate Analysis. Journal of Statistical Software, 25(1), 1-18. https://doi.org/10.18637/jss.v025.i01

Montero, L., \& García, J. (2017). Panorama multidimensional del desarrollo urbano en América Latina y el Caribe. Recuperado en 29 de junio de 2021 de https://repositorio.cepal.org//handle/11362/41974

Nathaniel, S. P., Nwulu, N., \& Bekun, F. (2020). Natural resource, globalization, urbanization, human capital, and environmental degradation in Latin American and Caribbean countries. Environmental Science and Pollution Research, 115. https://doi.org/10.1007/s11356-020-10850-9

Pérez, C. (2004). Técnicas de análisis multivariante de datos. Aplicaciones con SPSS. Madrid: Pearson Prentice Hall.

Permentier, M., Bolt, G., \& Ham, M. van. (2010). Determinants of Neighbourhood Satisfaction and Perception of Neighbourhood Reputation. Urban Studies, 48(5), 977-996. https://doi.org/10.1177/0042098010367860 
Ramkissoon, H., Smith, L. D. G., \& Weiler, B. (2013). Relationships between place attachment, place satisfaction and proenvironmental behaviour in an Australian national park. Journal of Sustainable Tourism, 21(3), 434-457.

https://doi.org/10.1080/09669582.2012.708042

Reichenbach, H. (2006). Experience and Prediction. Recuperado en 29 de junio de 2021 de https://undpress.nd.edu/9780268040550/experience-and-prediction/

Reid, L., Sutton, P., \& Hunter, C. (2010). Theorizing the meso level: The household as a crucible of pro-environmental behaviour. Progress in Human Geography, 34(3), 309-327. https://doi.org/10.1177/0309132509346994

Rodríguez, J. (2008). Movilidad cotidiana, desigualdad social y segregación residencial en cuatro metrópolis de América Latina. Eure, 34(103), 49-71. https://doi.org/10.4067/s0250-71612008000300003

Ruiz-Tagle, J. (2015). La persistencia de la segregación y la desigualdad en barrios socialmente diversos: un estudio de caso en La Florida, Santiago. Revista EURE - Revista de Estudios Urbano Regionales, 42(125). Recuperado en 29 de junio de 2021 de http://www.eure.cl/index.php/eure/article/view/1330

Saraví, G. A. (2008). Mundos aislados: Segregación urbana y desigualdad en la ciudad de México. Eure, 34(103), 93-110. https://doi.org/10.4067/s0250-71612008000300005

Stern, P., Dietz, T., Abel, T., Guagnano, G., \& Kalof, L. (1999). A Value-Belief-Norm Theory of Support for Social Movements: The Case of Environmentalism. Human Ecology Review, 6(2). Recuperado en 29 de junio de 2021 de https://cedar.wwu.edu/hcop_facpubs/1

Thibert, J., \& Osorio, G. A. (2014). Urban Segregation and Metropolitics in Latin America: The Case of Bogotá, Colombia. International Journal of Urban and Regional Research, 38(4), 1319-1343. https://doi.org/10.1111/1468-2427.12021

Tosoni, M. (2011). Efectos del Barrio La Gloria: Experiencias del lugar y estrategias simbólicas de los sectores populares en el Gran Mendoza. Cuadernos de Antropología Social, (34), 29-50. https://doi.org/10.34096/cas.i34.1378

Xu, J., \& Han, R. (2019). The Influence of Place Attachment on Pro-Environmental Behaviors: The Moderating Effect of Social Media. International Journal of Environmental Research and Public Health, 16(24), 5100. https://doi.org/10.3390/ijerph16245100

Yunda, J. G. (2019). Densificación y estratificación social en Bogotá: distribución sesgada de la inversión privada. Revista EURE - Revista de Estudios Urbano Regionales, 45(134). Recuperado en 29 de junio de 2021 de http://www.eure.cl/index.php/eure/article/view/2665

Editor: Rodrigo Firmino

Recibido: 16 feb. 2021

Aprobado: 17 sep. 2021 


\section{Errata}

En el artículo "Segregación espacial, satisfacción con el barrio y comportamientos responsables con el medio ambiente en una ciudad latinoamericana", con el número de DOI https://doi.org/10.1590/2175-3369.013.e20210043, publicado en urbe. Revista Brasileira de Gestão Urbana, vol. 13, e20210043, en la página 1,

donde lees:

"Paola García Reyes"

leer:

"Paola García-Reyes"

donde lees:

"Natalia Andrea Hernández Vargas"

leer:

"Natalia Andrea-Hernández-Vargas"

donde lees:

"Ciro David Murillo Lenis"

leer:

“Ciro David Murillo-Lenis"

donde lees:

"Cómo citar: Reyes, P. G., Vargas, N. A. H., \& Lenis, C. D. M. (2021). Segregación espacial, satisfacción con el barrio y comportamientos responsables con el medio ambiente en una ciudad latinoamericana. urbe. Revista Brasileira de Gestão Urbana, v. 13, e20210043.

https://doi.org/10.1590/2175-3369.013.e20210043"

leer:

“García-Reyes, P., Hernández-Vargas, N. A., \& Murillo-Lenis, C. D. (2021). Segregación espacial, satisfacción con el barrio y comportamientos responsables con el medio ambiente en una ciudad latinoamericana. urbe. Revista Brasileira de Gestão Urbana, v. 13, e20210043.

https://doi.org/10.1590/2175-3369.013.e20210043" 